\title{
Cross-Cultural Similarities and Differences in Person-Body Reasoning: Experimental Evidence From the United Kingdom and Brazilian Amazon
}

\author{
Emma Cohen, ${ }^{\text {a,b,c }}$ Emily Burdett, ${ }^{\mathrm{c}}$ Nicola Knight, ${ }^{\mathrm{c}, *}$ Justin Barrett ${ }^{\mathrm{c}}$ \\ ${ }^{\mathrm{a}}$ Max Planck Institute for Psycholinguistics, Nijmegen \\ ${ }^{\mathrm{b}}$ Max Planck Institute for Evolutionary Anthropology, Leipzig \\ ${ }^{\mathrm{c}}$ Institute of Cognitive and Evolutionary Anthropology, University of Oxford
}

Received 18 December 2009; received in revised form 30 October 2010; accepted 10 November 2010

\begin{abstract}
We report the results of a cross-cultural investigation of person-body reasoning in the United Kingdom and northern Brazilian Amazon (Marajó Island). The study provides evidence that directly bears upon divergent theoretical claims in cognitive psychology and anthropology, respectively, on the cognitive origins and cross-cultural incidence of mind-body dualism. In a novel reasoning task, we found that participants across the two sample populations parsed a wide range of capacities similarly in terms of the capacities' perceived anchoring to bodily function. Patterns of reasoning concerning the respective roles of physical and biological properties in sustaining various capacities did vary between sample populations, however. Further, the data challenge prior ad-hoc categorizations in the empirical literature on the developmental origins of and cognitive constraints on psycho-physical reasoning (e.g., in afterlife concepts). We suggest cross-culturally validated categories of "Body Dependent" and "Body Independent" items for future developmental and cross-cultural research in this emerging area.
\end{abstract}

Keywords: Mind-body; Dualism; Person; Cognitive anthropology; Cross-cultural; Brazil

\section{Introduction}

Recently, there has been growing interest among cognitive psychologists and sociocultural anthropologists in the developmental origins and cross-cultural incidence of mind-body dualism (e.g., Bloom, 2004; Cohen, 2008; Hodge, 2008; Wellman \& Johnson,

\footnotetext{
*Dr. Nicola Knight died in June 2009 as this paper was in preparation.

Correspondence should be sent to Emma Cohen, Max Planck Institute for Psycholinguistics, Wuntlaan 1, Nijmegen, Netherlands. E-mail: emma_cohen@eva.mpg.de
} 
2008). Some developmental psychologists have hypothesized that the autonomy of cognitive systems dealing with the social and physical worlds renders naïve, or intuitive, psycho-physical dualism a default cognitive stance in reasoning about human and humanlike agents (Bloom, 2004; Wellman \& Johnson, 2008; Wynn, 2008). Two main strands of inquiry bear upon this position.

The first strand investigates children's and adults' reasoning about phenomena that entail interactions across physical/biological and psychological domains (e.g., psychogenic bodily events). Evidence suggests that reasoning about cross-domain psycho-physical interactions presents conceptual challenges (e.g., Hatano \& Inagaki, 1994; Inagaki \& Hatano, 1993; Notaro, Gelman, \& Zimmerman, 2001, 2002; Schultz, Bonawitz, \& Griffiths, 2007). North American children, for instance, had difficulty appreciating the possibility of worry-induced stomach ache or fear-induced goosebumps (Notaro et al., 2001, 2002). Some researchers have suggested that the domain distinction that enables children to distinguish appropriately between mental events and their physical counterparts (e.g., a thought about a house is different from an actual house; wanting to get taller is different from actually getting taller) constrains reasoning about phenomena that entail causal interactions across domains (Wellman \& Johnson, 2008).

Further research has shown that under certain conditions, such as when evidence of cross-domain causes and effects are presented (e.g., a fear-induced tummy ache), 4 and 5 year olds (but not younger children) can entertain this as a causal possibility, even though it conflicts with their prior beliefs about domain-appropriate causes (Schultz et al., 2007).

Despite developmental changes, however, there is some evidence to suggest that a bias toward within-domain causal attribution is not easily revised or overridden. Although adults display little difficulty in reflectively appreciating the possibility of cross-domain interactions (as demonstrated in some of the above studies), recent studies among clinical professionals have shown a tendency to dichotomize mental and biological causes and cures when reasoning about clinical phenomena (Ahn, Proctor, \& Flanagan, 2009; Miresco \& Kirmayer, 2006). Furthermore, participants differentially attributed responsibility for illness-related behaviors depending on whether they subjectively evaluated the illness as having a psychological or biological etiology (Miresco \& Kirmayer, 2006). This bias appears to persist alongside the contrary, dominant mind-body integrative position that characterizes modern medical science and professional training, suggesting divergence between explicit and implicit theories. One discussion concludes that "similarities between expert clinicians and laypeople could reflect deep-seated inclinations to separate biology from psychology" (Ahn et al., 2009, p. 172).

The second strand of relevant research investigates reasoning about phenomena that entail the conceptual unbuckling of mind and body. Recently, several studies have investigated children's understandings of biological death and, in particular, their expectations about the post-death continuity or discontinuity of mental states (Astuti \& Harris, 2008; Bering \& Bjorklund, 2004; Bering, Hernández Blasi, \& Bjorklund, 2005; Harris \& Giménez, 2005). Research with American children suggests a developmental trend that is contingent upon developing understandings of interactions between psychological and biological domains. Specifically, the age-related decrease in children's tendency to judge 
psychological traits as continuing following death (continuity judgements) is attributed to children's developing knowledge of biological function and its supporting role in psychological function (Bering \& Bjorklund, 2004).

Bering and Bjorklund (2004) argue that, as biological knowledge develops, the newly acquired appreciation that mental capacities are contingent upon biological function is applied to reasoning about the mental capacities of dead agents. Finer-grained analysis of different categories of mental state-distinguished according to the degree to which states "conceptually at least, appear planted in the body" (Bering \& Bjorklund, 2004, p. 218) further supports this interpretation of children's reasoning patterns. Compared with questions about psychobiological states, for example, questions concerning epistemic mental states (e.g., knowing, remembering) were more likely to elicit continuity judgements among late elementary children and adults.

Cross-cultural research in Spain and Madagascar has shown that response patterns are influenced by the ways in which narratives about death are framed (Astuti \& Harris, 2008; Harris \& Giménez, 2005). For example, when death is described in a biomedical context, such as in a hospital, continuity judgements are elicited less frequently than when death is described in the context of funerary rituals that imply the continuity of the person in some form. Although the research programs investigating the development of afterlife concepts are methodologically divergent (e.g., in age-groups investigated, questionnaire composition, response options, and coding practices), and therefore offer results of limited comparability, together they suggest that concepts that entail psychological continuity following biological death are readily acquired and used. Such concepts do not appear to encounter the kind of cognitive impediment apparent in reasoning about cross-domain psycho-physical/biological interactions.

Some recent research with infants has sought to investigate more directly the developmental origins of this facility to reason about the autonomy of mental phenomena from bodily function. Kuhlmeier, Bloom, and Wynn (2004a) present controversial but suggestive findings that 5-month-old infants do not apply the principle of continuous motion to humans, suggesting that "infants do not readily view humans as material objects" (p. 101) and that humans' agentive properties are so salient to the infant participants as to eclipse the presence of their physical properties (see also Kuhlmeier, Bloom, \& Wynn, 2004b; Rakison \& Cicchino, 2004; Saxe, Tzelnic, \& Carey, 2006; Wynn, 2008).

Although there is still limited experimental data bearing directly on the question of whether, at least for some mental and bodily events under some conditions, mind-body autonomy is a default intuitive stance, these findings jointly suggest that: (a) the capacity to distinguish between psychological and physical causation emerges early in development, and concepts that entail a conceptual distinction between these two domains are readily acquired and used; (b) this receptiveness to dualism may be attributable to an early developing discrimination between mental and physical events that constrains children's reasoning about psychogenic bodily events and illnesses in cases where it is both appropriate and inappropriate to do so; (c) this bias persists, at least for some domains and under some conditions, into adulthood-widespread concepts of death, illness, invisible companions, supernatural entities, and brain, mind, and identity, also appear to be underpinned by the 
capacity readily to conceive of persons and their bodies as separable (e.g., Cohen, 2008; Cohen \& Barrett, 2008a,b).

Ethnographic data on the cross-cultural recurrence of dualistic concepts of afterlife, deities, spirit possession, reincarnation, illness, and so on are frequently drawn upon to support the claim that psycho-physical dualism is pervasive in reasoning about agents and things (e.g., Bloom, 2004; Cohen, 2008). Yet psychological and anthropological research programs have proceeded along divergent trajectories, arriving at incommensurable theoretical positions. Despite the huge scope for theoretical and methodological crossfertilization, interdisciplinary dialog and debate has been slow to develop (but see Astuti, 2001).

Anthropologists, on the whole, no longer dispute the claim that the dualistic separation of mind from body is a historical and cultural construction. According to this view, mind-body dualism is culturally localized, rooted in a particularly influential philosophy-namely Cartesianism - and is "but one among many systems of knowledge regarding relations held to obtain between mind [and] body" (Scheper-Hughes \& Lock, 1987, p. 11), and "many (if not most) non-Western people ... simply do not recognize anything comparable to the social/biological distinction as articulated in western discourse" (Ingold, 1991, p. 362; see also Strathern, 1996; Lambek, 1998).

Ethnographic evidence brought to bear on these claims is often difficult to interpret, however. Empirical claims and data are frequently couched in abstruse metaphorical language (e.g., "Gahuku notions do not parallel, but collapse, western mind/body categories. For them ... the body swallows and contains the mind, and also becomes itself the mark of the person"' [Strathern, 1994, p. 45]). Sources of evidence, and their quality and representativeness, are also frequently obscure. Nevertheless, relevant data suggest considerable crosscultural variability in the ways in which "mind" and "body" and interactions between them are construed. The Cashinahua of the Peruvian and Brazilian Amazon, for example, construe knowledge as "an intimate part of each developing body" (McCallum, 1996, p. 355) and certain organs, such as the skin, hands, ears, genitals, liver, and eyes, are believed to be linked to processes of learning in a physical sense. Skin knowledge, for example, is said to be actually attached to the skin. Disturbances in bodily function can thus have "dire consequences" for knowledge (ibid, p. 347). Such ideas about mind-body interdependencies are not dissimilar to Western understandings that brain function and impairment cause psychological disturbances or the view that heart and liver transplants, for example, can result in the transference of aspects of the donor's psychological character to the recipent. ${ }^{1}$

The ethnographic literature further presents widespread examples of psychogenic physical illness, and of instances in which one's illness is not caused by one's own thoughts but rather the thoughts of others. For example, in many parts of Melanesia, thoughts, actions, and attitudes of living and dead kinsfolk are believed to influence a person's health (e.g., Frankel, 1986; Lewis, 2004). Similarly, across Lowland South America, many people reason that biological ties between persons are not exclusively forged via biological kinship (e.g., Vilaça, 2002). The Huaorani of Ecuador, for example, affirm that people living in the same longhouse gradually become of the same substance-as they say, "of one same flesh.' (Rival, 1998, p. 621) The physical reality of living together, that is, of continuously 
feeding each other, eating the same food, and sleeping together, develops into a common biological constitution, which is "far more real" than geneaological ties (ibid.). While none of these examples supports the strong claim that non-Western populations are free of dualistic concepts and reasoning, they-and many others-point to considerable cross-cultural variability in the ways in which people conceptualize possible interactions between social, psychological, biological, and physical domains in the construction and constitution of the person.

The overall picture emerging from anthropological and psychological research suggests that there may be some aspects of experience about which people may readily reason in terms of the autonomy of mental and bodily domains, and some aspects about which people readily reason in terms of the interdependencies of mental and bodily domains. There is surprisingly little systematic cross-cultural and interdisciplinary research on these issues, however. Many questions remain as to where any strong intuitions and cognitive constraints lie, their origins and development, the conditions under which they are activated, and how they shape cultural expression and transmission. Systematic, cross-cultural data are needed to test claims about the cultural uniqueness of so-called Western mind-body dualism. Cognitive psychological research is required to investigate the ways in which cognitive function in these domains constrains and informs cultural uniformity and diversity.

In this paper, we present evidence that begins to address these questions and evidential gaps. We investigated and compared person-body reasoning in two culturally distinct populations, one in the United Kingdom and one in the northern Brazilian Amazon. Our aim was not to establish whether dualistic reasoning (or, perhaps more relevantly, biologicalphysical-psychological "trialism") is a default mode of reasoning. Rather we set out to explore whether the two populations exhibit any common intuitions about the relationships between certain traits and bodily properties. More specifically, using a novel interview task, we investigated reasoning about a broad range of basic psychological capacities and aptitudes and their perceived anchoring to the body, and specifically to physical and biological properties. The fundamental question was, "What do people intuitively consider to be left of themselves once they have been stripped of their bodies?', Participants reasoned about novel and hypothetical mind-body phenomena, such that any reflective theory they may have held about mind-body phenomena in general would provide little recourse for the generation of relevant inferences and judgements.

This exploratory investigation was carried out in Oxford, UK, and in the province of São Sebastião da Boa Vista, Marajó Island, northern Brazil. A brief demographic and ethnographic portrait of the Brazilian research site is given in the next section.

The study had three between-subjects conditions. Condition 1 investigated the contours of people's "disembodied person" concept. Participants were asked to imagine they have left their body and to offer judgements about the likely continuity or discontinuity of a range of capacities (e.g., seeing, knowing, feeling happy, etc.). By encouraging participants to reason about disembodied existence as though it were a literal possibility, we aimed to probe beneath explicit beliefs to implicit intuitions. Though participants may believe such an extraction from one's body to be impossible, this protocol would, nevertheless, reveal any converging intuitions regarding what particular capacities would or would not continue 
provided such a disembodiment were possible. If participants find such a body-person divorce difficult or impossible to contemplate even as a hypothetical scenario, we would not expect converging intuitions about which traits would or would not continue, but random or arbitrary answering. Convergent responses potentially suggest convergent intuitions, provided that participants have understood the task. Further, if person-body reasoning is wholly derived from socially transmitted culture, and mind-body dualism is a uniquely Cartesian heritage of Western populations, we would expect to see considerable divergence between any response patterns of the two sample populations.

In Conditions 2 and 3, participants were asked to imagine they have left their body and have entered a rock (C2) or plant (C3). Comparison of response patterns across conditions allowed us to investigate the relative importance of physical and biological properties in judgements about the continuity of various capacities. It seems likely that intuitions about bodily and mental phenomena are guided by a range of cognitive mechanisms, most fundamentally those having to do with psychology, physicality, and biology. The present study addresses the two-part question: (a) (how) does adding the property of physicality alter patterns of responses for certain items? and (b) does physicality alone alter response patterns, or does the presence of biological properties also influence people's judgements about the continuity of certain psychological capacities? Perhaps some psychological capacities are regarded as requiring a physical body whereas others require a biological, physical body. This method potentially allows identification of those traits considered to be anchored to greater or lesser degrees to physical, biological, or psychological function, or some combination of these properties. For the purposes of this initial investigation, "rock" was used as the target bounded physical object because rocks are familiar to both sample populations, and, as a natural objects, they do not import additional confounding properties that may be activated by artefacts (e.g., as intentionally crafted, and owned or associated with a particular person, or kind of person). "Plant" was selected as a relevant entity for the biological condition for its matched everyday familiarity with "rock" (used in the physical condition), and was chosen over "non-human animal" in order to avoid confounds that would potentially arise from the activation of intuitions about animal psychology.

Comparing results from all conditions cross-culturally, we aimed to explore regularities and differences across the patterns of responses between the UK and the Brazilian Amazon populations. The majority of participants in the Brazilian Amazonian community have had only minimal (1-3 years) or no formal schooling. Intuitive and reflective beliefs relevant to our interview task would have been almost exclusively honed through discourse about and direct experience of sickness and cure, for example, and of the natural biological and physical world of the river and forest within and from which they live. In contrast, UK participants have received scientific education in physics and biology (including biomedicine), and, against a traditional Cartesian cultural backdrop, increased exposure via popular neuroscience and neurology to the science of the brain and the brain-mind interface. Differences in formal and informal education about, and direct engagement with, divergent natural physical and biological environments may establish different intuitions about the respective roles of physicality and biology in sustaining 
various traits. Alternatively, fundamental regularities in participants' physical and biological environments and person-body experiences may sustain fairly uniform patterns of reasoning between populations about persons and bodies across the experimental conditions, with divergences in education, environment, and cultural tradition having little impact on intuitive expectations in this domain.

Finally, analyzing results from the Disembodied condition, we also sought to establish validated categories of "body dependent" and 'body independent'" states. Recent research on children's developing conceptions of psychological continuity after death has used

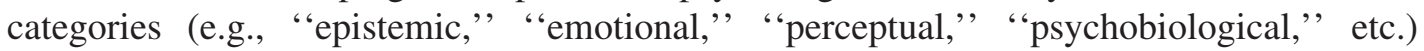
established according to predetermined assumptions about the degree to which the experimenters perceived psychological capacities, such as feeling hungry, remembering, seeing, etc., to be tied to biological function (e.g., Astuti \& Harris, 2008; Bering \& Bjorklund, 2004). The current study enabled assessment of the validity of these categories and the tentative establishment of cross-culturally validated categories for future research on "bodily" and "mental" capacities.

After a brief introduction to the Brazilian research setting, and description of the study design and participants, we present results and discussions in two blocks. First, we present and discuss the cross-cultural and cross-condition findings. We then assess the validity of trait categories used previously in the literature and suggest new categories for future research based on our findings. We finish with a general concluding discussion.

\section{Brazil research site: Marajo Island}

Marajó, meaning "barrier to the ocean," is a large archipelago $\left(40,000 \mathrm{~km}^{2}\right)$ at the Amazon River delta. The municipality of São Sebastião da Boa Vista (SSBV) is located at the south of the archipelago, just to the west of the confluence of the Pará and Tocantins Rivers. There is no published ethnographic research on this specific Brazilian community, but one of us (Cohen) has been conducting research in the broader region since 2002 (e.g., Cohen, 2007), including “interior' (or rural) regions of Pará and neighboring communities on Marajó Island. The research reported here was conducted during July-September 2008. A female assistant from Belém and anthropology graduate, whose mother and extended family are originally from SSBV, offered logistical support and advice on experimental implementation and participant recruitment, and delivered all questionnaires (while Cohen organized counterbalancing of questionnaires and noted responses and feedback). Two additional "Boa Vistense" locals (a female primary school teacher and a male assistant within the local government assembly offices) assisted with contacts and general local knowledge.

SSBV covers an area of 1,632 $\mathrm{km}^{2}$ and has a population of 20,500 (Instituto Brasileiro de Geografia e Estatistíca). There are three main towns, of which the municipal seat, also called São Sebastião da Boa Vista (abbrev. Boa Vista), is the largest. Research was mainly conducted in this town (population approximately 7,000). The town's main access from the state capital of Belém $(118 \mathrm{~km})$ is via an overnight ferry or barge due southwest through the 
Baía do Guajará, and along the Pará River, which borders the south side of the island. People live along the banks of the Pará River and its tributaries. Most households own or have ready access to some form of river transport, from dug-out canoes to fishing vessels of various sizes. In Boa Vista, the main strip of asphalted road surface doubles as a runway, which is used by air-ambulances as well as aircraft transporting politicians and other dignatories to and from the town.

Local subsistence-economic activities mainly include fishing and agro-extraction (mainly wood, bananas, açaí, palm, sugarcane, and beans). Livestock include pigs, hens, and a small number of cattle. Boa Vista is home to a palm heart processing plant, and it is at the heart of Brazil's açaí region. Services include a post office, schools, food market, clothing retailers, and a hardware store. The rising appeal of açaí products internationally has accelerated economic growth in the region. Marajó is one of the poorest regions of Pará state, however, contributing only $3 \%$ of the state GDP. According to the 2001 census, over half of the population of SSBV was under 18 years of age, and $82.7 \%$ of the population was under 40 years; $16.6 \%$ of the population over 10 years of age had minimal or no schooling, and $46 \%$ had obtained some primary school education or higher. The largest single group had 1-3 years of formal schooling.

There are no reliable census data on religious affiliation. Two main churches in Boa Vista - one Catholic and one Protestant_-were attended by a small subsection of the population. Ethnographic research, entailing informal conversation and interview with a broad section of the population and observation of church activities and attendance, suggests that, for the majority of our participants, formal religion was relatively unimportant in everyday life. Conversations on the subject tended to focus on local versions of widespread legends (lendas) and spirit-related religious practices (e.g., healing, possession, etc.). Although many explicitly expressed disbelief in such stories (e.g., of river dolphins, or botos, turning into charming men and seducing menstruating women), others were clearly influenced by at least the possibility of their truth (e.g., some women refrained from bathing in the river while menstruating). Government health provision of modern biomedical treatments has improved via the employment of community "health agents." Healing traditions derived from shamanistic curing practices, or cura, are still common in the prevention and treatment of illness. $^{2}$

Although local religious traditions suggest the presence of some form of person-body dualism (e.g., in concepts of spirit possession, soul flight, afterlife, etc.), it is possible that, following the claims of many anthropologists, this apparent dualism might be held reflectively as a wholly culturally transmitted principle and therefore would potentially be very different to Western forms, or confined to a narrow cultural domain that is of limited relevance to, or difficult to generalize to, the present study's novel scenario and questions. Alternatively, consistent and similar judgements across the populations that certain capacities could survive such a hypothetical disembodiment could potentially support the claim that the ability to reason in terms of person-body autonomy is, under certain conditions, present beyond the West, and the conceptual outputs of such reasoning are not wholly culturally transmitted (or a specifically Cartesian or Platonic legacy). 


\section{Methods}

\subsection{Participants}

In the UK, 147 people (97 women and 50 men) participated, mean age $=36$, range, 12-82 years. Random assignment to condition yielded 51 participants in the Disembodied Condition (mean age, 39), 48 in the Rock Condition (mean age, 34), and 48 in the Plant Condition (mean age, 37). Participants were recruited in an Oxford café. All participants received monetary compensation for time costs incurred (a 5 GBP coffee voucher, approx. US\$8). In Brazil, a total of 154 participants were recruited through word of mouth and face-to-face invitations (78 women and 76 men; mean age, 31; range, 14-78). There were 54 participants in the Disembodied Condition (mean age, 31), 49 in the Rock Condition (mean age, 32), and 51 in the Plant Condition (mean age, 30). All participants received monetary compensation of 5 BRL (approx. US\$3).

\subsection{Design and procedure}

The following brief framing narrative preceded a 51-item questionnaire (conditionrelevant amendments indicated in parentheses):

I want you to imagine that you leave your body [C1](and that you go into a rock [C2]/plant [C3]). You are not dead, but you have simply left your body (and gone into a rock [C2]/plant [C3]). Now, this might seem like a strange situation. But I want you to imagine that you can still exist once you have left your body. The questions that follow are about you, now that you have left your body (and are in a rock [C2]/plant [C3]).

The same series of questions, in counterbalanced order, were posed across all conditions. Questions followed the format, "Do you think that you can still do/feel, etc. X?', The 51 capacities included were as follows: want things, feel hungry, feel achy or sore, feel sexual desire, remember things, smell things, feel the wetness of the rain, imagine things, feel angry, feel thirsty, feel relaxed, hear things, feel excited, feel things touching you, feel scared, feel dirty (grubby), love things, feel faint, learn things, feel cold, feel lonely, feel dizzy, hate things, feel the blowing of the wind, feel surprised, feel sleepy, feel queasy, understand things, feel itchy, feel brave, feel upset, feel bloated, see things, feel pleased, watch things, feel hot, feel calm, feel the sun warming you, feel disgust, know things, feel happy, feel feverish, believe things, feel like going to the toilet, desire things, taste things, feel sad, have intentions, hope for things, listen to things, feel out of breath. ${ }^{3}$

Participants indicated their responses on a 6-point scale with anchors at each end indicating "definitely no", and "definitely yes." Each intermediate point was marked by a vertical line. UK participants read the questionnaire and marked responses on scales adjacent to each question.

In Brazil, the questionnaire, prepared via translation-backtranslation by bilingual English-Portuguese speakers who had lived in both Oxford and Belém, was delivered orally 
by a local first-language Brazilian Portuguese speaker. The research setting varied according to conditions (weather, noise disturbances from music or construction work on boats and piers, etc.) but tended to be a shaded outdoor or sheltered space with somewhere to sit out of earshot of passersby. The framing narrative was relayed to participants twice and any immediate requests for repetition/clarification addressed. The experimenter explained to each participant that she would ask a series of questions based on the imagined situation. An enlarged printed and laminated image of the scale was used and participants pointed to it to indicate their answers. The experimenter explained how the scale worked, drawing attention to the anchors at each end (e.g., "If your response is 'Definitely Yes,' point here') and the significance of the points in between (e.g., "If your response is 'No,' but not quite 'Definitely No,' you can point here'’). Participants indicated no difficulty understanding the instructions or using the scale during the exercise. Participants were given further opportunity to ask questions about the framing. Queries tended to be rhetorical and merely elicit repetition of the instructions; "So, I just have to imagine'”; "I've left my body"; " I'm not dead," etc. Following clarification, the experimenter confirmed whether the participant had understood and imagined the situation and asked if she could continue with a series of questions referring to the imagined situation. Participants responded swiftly and without hesitation to the questions. Each interview lasted approximately 10-12 min (4-5 min for instruction, 5-7 min for experimental questions and responses). Data from one male participant were excluded from analysis on grounds of alcohol intoxication.

General conversation and any spontaneous feedback on the exercise were reserved for the end of the session. Participants appeared to enjoy the exercise and, most important, their feedback indicated that they had imagined the situation as intended-some talked about how they imagined leaving their body and going into a sunflower, or being in a large rock, or going about "taking a turn" (dando uma volta) without their body, etc. People commonly seemed somewhat bemused as to why we might be interested in their answers to such questions and were curious to know what it was all about. The very low frequency with which participants drew a link to any potentially relevant cultural references suggests that framing narrative and questions were indeed novel and that reflective, elaborate cultural beliefs were unlikely drawn upon to inform responses.

\section{Results and discussion}

\subsection{Part 1: Cross-cultural and cross-condition comparisons}

No sex differences were detected in mean responses for the 51 items by sample and condition or in any further analyses reported, nor were any question-order effects detected.

We first analyzed responses from the Disembodied Condition, observing whether participants appeared to differentiate among items. The spread of mean responses showed this to be the case-in both samples, mean responses were sufficiently varied and spread over a sufficiently large range as to suggest that items were reasoned about differently (UK range, 2.26-5.24, interquartile range, 3.09-4.77; Brazil range, 2.39-5.44, interquartile range, 
3.21-4.77). One-sample $t$-tests revealed that 43 (UK) and 27 (Brazil) of the 51 items significantly deviated from the scale's midpoint (3.5).

To examine the agreement between the two samples' mean rankings of items, we conducted a Spearman's rank correlation, confirming a significant positive correlation (Spearman's $r=.861, p<.001$, two-tailed).

Next, we analyzed the effect of condition (Disembodied, Rock, Plant) on responses. For each sample separately, we collapsed all items that received a continuity score significantly greater than 3.5 in the Disembodied Condition into a single category (Body Independent) and combined all other items into a second category (Body Dependent). The Body Independent and Body Dependent categories for the UK sample contained 30 and 21 items, respectively, and for the Brazil sample, 22 and 29 items, respectively-see Table 1 for details.

All 22 items items in the Brazil Body Independent category were matched in the corresponding category for the UK. The eight non-matching items (i.e., in the UK Body Independent category but the Brazil Body Dependent category) were feel sexual desire, feel angry, feel excited, feel scared, hate things, feel upset, feel disgust, feel sad.

A 2 (sample) by 3 (condition) ANOva for the mean continuity scores of the Body Dependent category revealed significant main effects of condition and sample (Condition: $F(2$, $295)=7.823, p<.001$; Sample: $F(1,295)=18.309, p<.001)$. In the UK sample, pairwise comparisons (LSD corrected) showed a significantly higher mean response in the Plant Condition $(3.48, S D=0.90)$ compared with the Disembodied Condition $(2.87, S D=1.06$; $p=.009$ ). This result was replicated in the Brazil sample (Plant: $3.86, S D=1.18$, Disembodied: $3.30, S D=1.26 ; p=.012$ ), where there was also a significant difference between the Disembodied and the Rock Conditions (Rock: 4.02, $S D=1.31, p=.002$ ). Compared with the mean response in the Rock Condition in the UK sample $(3.14, S D=1.09)$, the mean response for Rock in the Brazil sample was significantly greater $(p<.001)$.

These results indicate that, whereas in the Brazil sample the introduction of physicality in the Rock Condition raises the mean of the Body Dependent category significantly, in the UK sample, mean scores increase significantly only when physicality and biology are combined.

A further $2 \times 3$ ANOVA compared responses across samples and conditions for the Body Independent category, revealing main effects of condition $(F[2,295]=9.559, p<.001)$ and sample $(F[1,295]=19.601, p<.001)$, and a significant condition-by-sample interaction effect $(F[2,295]=5.231, p=.006)$. Pairwise comparisons revealed no significant differences across conditions for the Brazil sample (Disembodied: 4.86, $S D=1.13$, Rock: 5.11, $S D=.81$, Plant: $4.85, S D=0.80)$. In the UK sample, the mean response for Plant $(3.89$, $S D=1.08)$ was significantly less than that of Disembodied $(4.73, S D=0.90)$ and Rock $(4.76, S D=0.85)$ and than that of the Plant condition in the Brazil sample (all $p<.001$; see Fig. 1).

These results suggest that, when asked to imagine leaving their bodies, and to reason about what they would still feel, perceive, know, and so on, participants in both samples could readily imagine the continuity of some capacities and the cessation of others. Such a task did not bewilder participants such that they resorted to uniform or random responding or idiosyncratic heuristics. Participants in both samples judged some capacities to be less 
Table 1

Mean scores and standard deviations for all items in the Disembodied condition

\begin{tabular}{|c|c|c|c|}
\hline UK & Mean $(S D)$ & Brazil & Mean $(S D)$ \\
\hline Remember things & $5.24(0.96)$ & Hope for things & $5.44(1.46)$ \\
\hline Watch things & $5.19(1.03)$ & Learn things & $5.44(1.49)$ \\
\hline See things & $5.14(1.21)$ & See things & $5.31(1.70)$ \\
\hline Imagine things & $5.11(1.11)$ & Know things & 5.24 (1.69) \\
\hline Know things & $5.06(1.06)$ & Feel brave & $5.22(1.72)$ \\
\hline Love things & $5.06(1.09)$ & Feel happy & $5.13(1.87)$ \\
\hline Understand things & $4.98(1.28)$ & Believe things & 5.07 (1.96) \\
\hline Hear things & $4.89(1.23)$ & Desire things & $5.04(1.92)$ \\
\hline Want things & $4.89(1.22)$ & Understand things & $4.98(1.96)$ \\
\hline Feel happy & $4.88(1.00)$ & Want things & 4.94 (1.96) \\
\hline Believe things & $4.82(1.13)$ & Listen to things & $4.91(2.03)$ \\
\hline Feel pleased & $4.79(1.250$ & Feel Calm & $4.81(2.09)$ \\
\hline Listen to things & $4.77(1.33)$ & Remember things & $4.80(2.10)$ \\
\hline Hope for things & $4.77(1.34)$ & Watch things & $4.76(2.15)$ \\
\hline Feel lonely & $4.76(1.38)$ & Feel surprised & $4.72(2.14)$ \\
\hline Feel brave & $4.75(1.21)$ & Imagine things & $4.70(2.14)$ \\
\hline Learn things & $4.70(1.48)$ & Love things & $4.57(2.20)$ \\
\hline Feel calm & $4.70(1.31)$ & Hear things & $4.54(2.25)$ \\
\hline Feel sad & $4.69(1.25)$ & Have intentions & $4.46(2.23)$ \\
\hline Feel relaxed & $4.66(1.30)$ & Feel relaxed & $4.41(2.31)$ \\
\hline Feel upset & $4.65(1.37)$ & Feel lonely & $4.35(2.32)$ \\
\hline Desire things & $4.58(1.36)$ & Feel pleased & $4.15(2.33)$ \\
\hline Have intentions & $4.57(1.32)$ & Feel the wind & $4.02(2.35)$ \\
\hline Feel angry & $4.54(1.52)$ & Feel upset & $4.00(2.36)$ \\
\hline Feel surprised & $4.51(1.38)$ & Feel sad & $3.89(2.42)$ \\
\hline Feel excited & $4.39(1.51)$ & Feel sexual desire & $3.81(2.45)$ \\
\hline Hate things & $4.32(1.59)$ & Feel the sun & $3.80(2.41)$ \\
\hline Feel sexual desire & $4.13(1.63)$ & Feel hot & $3.74(2.43)$ \\
\hline Feel scared & $4.13(1.57)$ & Feel sleepy & $3.74(2.46)$ \\
\hline Feel disgust & $4.11(1.48)$ & Smell things & $3.70(2.44)$ \\
\hline Smell things & $3.63(1.69)$ & Feel cold & $3.63(2.48)$ \\
\hline Feel sleepy & $3.51(1.77)$ & Hate things & $3.59(2.41)$ \\
\hline Feel the sun & $3.40(1.67)$ & Feel thirsty & $3.59(2.49)$ \\
\hline Feel the wetness of the rain & $3.18(1.84)$ & Feel touch & $3.56(2.45)$ \\
\hline Feel the wind & $3.12(1.66)$ & Feel faint & $3.43(2.46)$ \\
\hline Feel cold & $3.11(1.60)$ & Feel the wetness of the rain & $3.30(2.40)$ \\
\hline Feel hot & $3.10(1.54)$ & Feel dizzy & $3.24(2.41)$ \\
\hline Taste things & $3.09(1.56)$ & Taste things & $3.22(2.44)$ \\
\hline Feel dizzy & $3.09(1.59)$ & Feel angry & $3.20(2.44)$ \\
\hline Feel thirsty & $3.04(1.59)$ & Feel excited & $3.20(2.47)$ \\
\hline Feel touch & $2.93(1.58)$ & Feel the need to go to the toilet & $3.20(2.41)$ \\
\hline Feel dirty & $2.73(1.46)$ & Feel hungry & $3.15(2.44)$ \\
\hline Feel hungry & $2.64(1.56)$ & Feel out of breath & $3.06(2.39)$ \\
\hline Feel faint & $2.58(1.50)$ & Feel scared & $3.04(2.48)$ \\
\hline Feel achy/sore & $2.57(1.36)$ & Feel achy/sore & $3.00(2.41)$ \\
\hline
\end{tabular}


Table 1

Continued

\begin{tabular}{llll}
\hline UK & Mean $(S D)$ & Brazil & Mean (SD) \\
\hline Feel queasy & $2.55(1.32)$ & Feel disgust & $2.98(2.45)$ \\
Feel feverish & $2.51(1.42)$ & Feel itchy & $2.72(2.31)$ \\
Feel bloated & $2.40(1.40)$ & Feel queasy & $2.56(2.25)$ \\
Feel itchy & $2.39(1.33)$ & Feel bloated & $2.46(2.13)$ \\
Feel the need to go to the toilet & $2.28(1.46)$ & Feel feverish & $2.46(2.24)$ \\
Feel the sun & $2.26(1.28)$ & Feel dirty & $2.39(2.26)$ \\
\hline
\end{tabular}

Note: Items are organized by category and sample (in order of descending mean score). Body Independent items (i.e., items with a mean score significantly greater than 3.5) are shown in regular font; Body Dependent items (all other items) are shown in italics.

likely to continue than others, suggesting that they considered some capacities to be more strongly anchored to bodily function than others. Further, there was significant intersample agreement in the items receiving discontinuity and continuity judgements.

Note, however, that although participants judged some capacities to be strongly body independent and some to be strongly body dependent, several items elicited weak continuity and weak discontinuity scores. This suggests that certain capacities are perceived not to be primarily dependent on either person or body, but rather they are thought to be dependent to some (perhaps differing) degree on both. Alternatively, for some capacities, participants may have had very weak or widely divergent intuitions resulting in these middling scores. In either case, we suggest that the ease with which people reason about the autonomy of body and mind is likely capacity-contingent and context-dependent rather than an inevitable
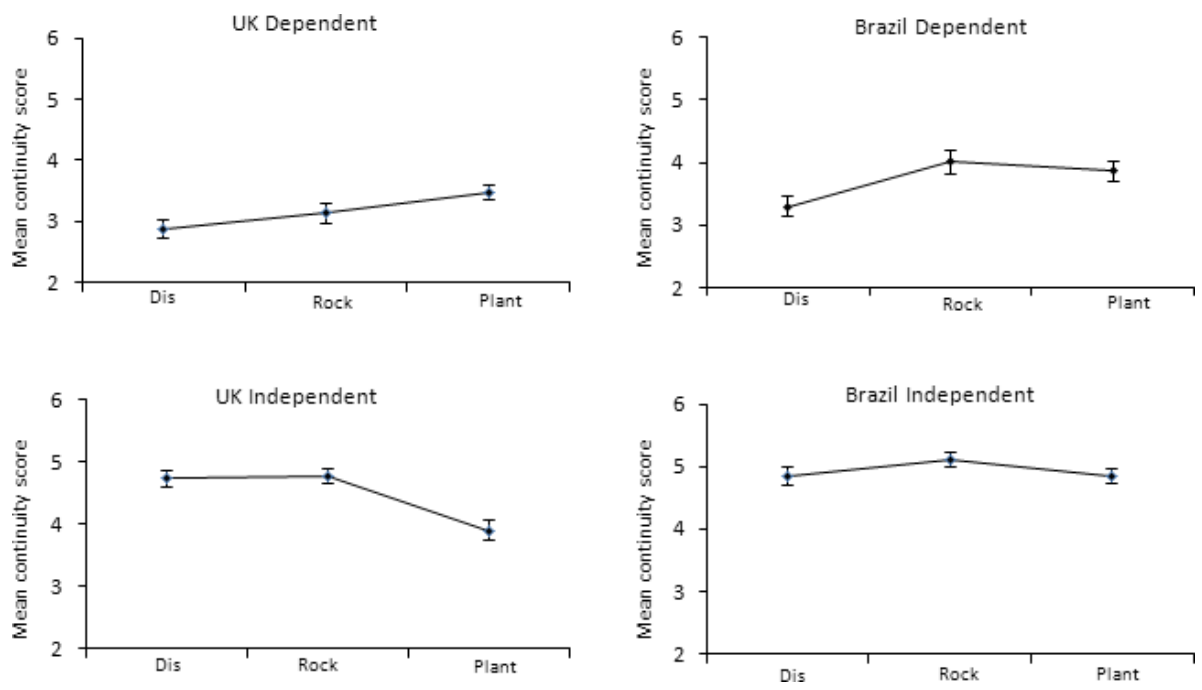

Fig. 1. Mean continuity scores for Body Dependent and Body Independent items by condition and sample. 
default across the board. The radical unbuckling of perceived mental and bodily contributions to a capacity's continuity may, under certain conditions, meet with conceptual difficulty.

The results further showed interesting cross-condition and cross-cultural differences. For cross-condition analyses, we separated Body Independent items (those receiving a mean score significantly higher than the midpoint of the scale, or 3.5, in the Disembodied Condition) from Body Dependent items (all remaining items). In both samples, Body Dependent items obtained higher mean scores in the Rock and Plant Conditions than in the Disembodied Condition. That is, capacities were regarded as more likely to continue when some kind of body was available than if there were none at all. In Brazil, specifically when participants reasoned about leaving their body and going into a rock, mean scores for Body Dependent items rose significantly. Adding the property of biology (in the Plant Condition) had no effect on these items. For UK participants, however, adding physicality raised scores for the Body Dependent category, but this category only achieved a significantly higher mean response when biology was further introduced.

This trend in UK responses is in line with the prediction that both physicality and biology are important in reasoning about bodily function and, by extension, interactions between bodily and mental functions. Human bodies are not just solid objects that obey principles of cohesion, solidity, gravity, and so on. By preschool age, children show an understanding of bodies as animate biological entities that possess parts that relate to movement and biological function, such as growth and illness (e.g., Hatano \& Inagaki, 1994). This role for biological function is apparent in the response patterns pertaining to the UK population in the present study. It is unclear why responses in the Brazil sample did not display a similar pattern. It appears that Body Dependent capacities were perceived to be dependent largely on physical properties. This would explain why there was a significant jump in mean response for the Body Dependent category between the Disembodied and Rock Conditions, but no further rise between the Rock and the Plant Conditions. The results suggest that although the samples were broadly in agreement regarding which capacities are more and less dependent upon the body, they differed on whether exclusively physical or physical-plus-biological function mattered for continuity. Follow-up research is required to investigate this possibility, and to identify whether indeed there are other psychological capacities, states, or aptitudes that were not included in the present questionnaire that the Brazilian population may perceive to have a significant biological dimension. Subsequent ethnographic research in Marajó (by Cohen), involving in-depth conversation with a range of people (of different ages and occupations, including fishermen, farmers, teachers, and housewives) on the biological and physical qualities of rocks and plants, and of a range of items from the original questionnaire, and on the possible existence of any relevant local legend or cultural belief about rocks, plants, and so on, have yielded no data relevant to explaining the cross-cultural differences (see below for further discussion).

Cross-condition comparisons of the Body Independent category of items revealed an intriguing result. While there were no differences across the three conditions in the Brazilian sample, the mean continuity response for the Body Independent category fell significantly in the Plant Condition in the UK sample. Although the mean score was still significantly 
greater than the midpoint of the scale (one-sample $t$-test: $M=3.89, \quad S D=1.08$, $t(47)=2.501, p=.016$ ) and therefore still well within the continuity range of the scale, the cross-condition comparison suggests a significant role for biological function in the perceived continuity of items that were judged to be strongly continuous in the Disembodied Condition. The reason for this response pattern is not clear, but one possible interpretation (discussed in more detail below) is that when certain aspects of biology are made salient to the UK population, they are also readily perceived as relevant to the functioning of certain capacities. Limited to the biology of a plant, these properties are judged to be inadequate to support specifically full-blown human psychological capacities. In effect, it appears that UK participants were more willing to grant continuity for strongly mental capacities to an entirely disembodied person than to a person with some biological properties.

This finding raises a number of interesting possibilities that merit follow-up attention. Our starting point concerns the basic question of how people represent disembodiment, for example, of self, other, ancestors, spirits, etc. Are spirits, for example, actually thought of as embodied? It may be that people can readily reflect upon the possibility of leaving the body, or of spirits as lacking bodies, but there is no research to date directly addressing the question of whether tacit representations of these entities tend to entail at least some implicit expectations concerning spatiality (e.g., do they occupy spatial form? do they cohere? etc.), physicality, and biology. Ethnographic evidence may be suggestive in this regard-ideas about disembodied entities who wage physical wars, get hungry, feel lonely and isolated, move in or out of earshot, and so on are far from uncommon.

A cross-condition comparison of the single item "to see things" is potentially informative also. This capacity ranked highly in the Disembodied Condition in both samples (achieving second and third mean rank in UK and Brazil, respectively). Mean scores for this item differed significantly across the conditions, however (Kruskal-Wallis H; UK: $\chi^{2}=18.173, d f=2, p<.001$; Brazil: $\left.\chi^{2}=7.564, d f=2, p=.023\right){ }^{4}$ One interpretation suggested by these results is that the disembodied person's absence of eyes and relevant biological mechanisms is not considered unless more specific attention is drawn to this fact. In the disembodied condition, the generic notion of an absent body appears not to activate the more specific consideration that the disembodied person now lacks visual perception mechanisms. When this absence is not made salient, seeing is perceived by participants as something they do, not their body. However, when the absence (in a rock or plant) of visual mechanisms is made more directly evident, participants incorporate this consideration into their continuity judgements.

Of course, in the Brazil sample, the mean response for the Body Independent category as a whole did not display comparable sensitivity to the introduction of biological properties and we must therefore be careful not to generalize the import of this finding or interpretation. Nevertheless, we suggest that in the Plant Condition, biological properties were made salient to the UK participants, and these properties were then readily judged as significantly influencing the likely continuity of certain psychological capacities for which specifically plant biology was perceived to be inadequate. This was the case not only for seeing, as demonstrated above, but for the Body Independent category as a whole. Simultaneously, compared with the sample in the Disembodied Condition, this sample judged items in the 
Body Dependent category to be more likely to continue. In the Brazilian sample, in contrast, although aspects of biological function were made salient in exactly the same way, they were not perceived to be relevant to continuity judgements for either the Body Dependent or Body Independent items. We propose that the apparent link from salience to relevance displayed for Body Independent items in the UK-Plant Condition suggests that the UK population more readily reasoned in terms of the dependency between biological and psychological domains than the Brazil population. This interpretation runs contrary to received wisdom that, in the context of world cultures, Western populations are uniquely and persistently resistant to reasoning about mental and bodily phenomena as mutually dependent. Indeed, the results suggest that the Brazilian population was comparatively less sensitive to the possibility of cross-domain interdependencies, and therefore, in the context of the current study, tended to reason more dualistically.

\subsection{Part 2: Validation of variegated state categories}

Recent research into children's developing reasoning about afterlife reports resistance to reasoning about the mental states of dead agents as discontinuous. Variegated mental state categories (e.g., psychobiological, perceptual, epistemic, etc.) have been used to explore the nature of this resistance. Although criteria for the establishment of categories have never been explicitly itemized, the degree to which states are perceived to be anchored to biological function seems to be of particular importance (see Bering \& Bjorklund, 2004, pp. 228-230). The validity of these categories has never been tested, however. Does continuity on one item of each category predict continuity of other items in the category or do participants regard some members of each category as continuous and others as discontinuous? To test the hypothesis that the items in each category were ranked similarly in terms of their anchoring to bodily function, we conducted Friedman tests for each of the categories from Bering \& Bjorklund, 2002 and Astuti \& Harris, 2008 for which we had sufficient data (i.e., on three or more items from the category). Data from the Disembodied condition only were analyzed. Results are reported in Table 2.

These analyses revealed significant within-category differences across all categories except for Epistemic in the Brazil sample, suggesting that intracategory items are not reasoned about similarly in terms of their perceived dependency upon biological function.

We suggest two revised categories of mental states based on mean rankings and intersample agreement in the current study: (a) Body Independent: see, hope, know, learn, feel happy, understand; (b) Body Dependent: feel bloated, feel queasy, feel dirty, feel itchy, feel feverish, feel achy/sore.

Categories were established by averaging mean rankings (standardized to a 0-1 scale) for each item across the two samples (data from the Disembodied condition only). All those items for which the intersample difference between rankings was greater than the average intersample difference $(0.15)$ were eliminated. The top six ranking items remaining were selected for inclusion in the Body Independent category and the bottom six were selected for inclusion in the Body Dependent category. Friedman test results and suggestively strong Cronbach's alphas per sample for each category are presented in Table 3. Follow-up 
Table 2

Friedman test statistics and Cronbach's alpha for variegated state categories from Bering and Bjorklund (2002) and Astuti and Harris (2008) $)^{\mathrm{a}}$

\begin{tabular}{|c|c|c|c|c|c|c|c|c|}
\hline & \multicolumn{4}{|c|}{ UK } & \multicolumn{4}{|c|}{ Brazil } \\
\hline & $\mathrm{n}$; mean $(S D)$ & $\chi^{2}$ & $d f$ & $p$ & $\mathrm{n} ;$ mean $(S D)$ & $\chi^{2}$ & $d f$ & $p$ \\
\hline \multicolumn{9}{|c|}{ Bering \& Bjorklund (2002) } \\
\hline Psychobiological $^{1}$ & $49 ; 2.93(1.56)$ & 12.64 & 3 & .005 & $54 ; 3.26(2.41)$ & 10.93 & 3 & .012 \\
\hline Perceptual $^{2}$ & $50 ; 4.19(1.42)$ & 56.61 & 3 & $<.001$ & $54 ; 4.19(2.16)$ & 36.69 & 3 & $<.001$ \\
\hline Emotional $^{3}$ & $49 ; 4.60(1.36)$ & 16.53 & 3 & $<.001$ & $54 ; 3.67(2.39)$ & 16.50 & 3 & .001 \\
\hline Epistemic $^{4}$ & $50 ; 5.04(0.76)$ & 7.62 & 2 & .022 & $54 ; 5.05(1.92)$ & 1.30 & 2 & .521 \\
\hline \multicolumn{9}{|c|}{ Astuti and Harris (2008) } \\
\hline Mental $^{5}$ & $50 ; 4.34(1.27)$ & 128.64 & 5 & $<.001$ & $54 ; 4.44(2.11)$ & 56.41 & 5 & $<.001$ \\
\hline Psychobiological $^{6}$ & $50 ; 4.22(1.33)$ & 64.09 & 2 & $<.001$ & $54 ; 4.33(2.13)$ & 30.77 & 2 & $<.001$ \\
\hline
\end{tabular}

${ }^{\mathrm{a}}$ Items included from present dataset: ${ }^{1}$ queasy, hungry, thirsty, sleepy; ${ }^{2}$ taste, hear, smell, see; ${ }^{3}$ scared, angry, sad, love; ${ }^{4}$ believe, know, remember; ${ }^{5}$ hungry, cold, know, hear, see, remember; ${ }^{6}$ see, hear, hungry.

Table 3

Friedman test statistics and Cronbach's alpha for Body Independent and Body Dependent categories

\begin{tabular}{|c|c|c|c|c|c|c|c|c|c|c|}
\hline & \multicolumn{5}{|c|}{ UK } & \multicolumn{5}{|c|}{ Brazil } \\
\hline & $\begin{array}{l}\mathrm{n} ; \text { mean } \\
(S D)\end{array}$ & $\chi^{2}$ & $d f$ & $p$ & $\begin{array}{c}\text { Cronbach's } \\
\text { alpha }\end{array}$ & $\mathrm{n} ;$ mean $(S D)$ & $\chi^{2}$ & $d f$ & $p$ & $\begin{array}{c}\text { Cronbach's } \\
\text { alpha }\end{array}$ \\
\hline Body Independent & $48 ; 4.92(1.23)$ & 7.64 & 5 & .177 & .87 & $54 ; 5.26(1.7)$ & 4.09 & 5 & .537 & .73 \\
\hline Body Dependent & $48 ; 2.53(1.38)$ & 7.54 & 5 & .184 & .87 & $54 ; 2.6(2.27)$ & 4.64 & 5 & .461 & .72 \\
\hline
\end{tabular}

research with larger sample sizes would enable further exploratory factor analysis across all items for the identification of finer-grained categorical dimensions.

These findings do not necessarily invalidate the interesting intercategory differences detected in previous studies, but rather suggest that more accurate categories, supported by relevant data rather than developed according to a priori assumptions of the experimenter, might yield more meaningful results. For example, our findings suggest that it is inappropriate to jointly analyze reasoning about the post-death continuity of the capacities to see, hear, and feel hungry (as in the Astuti \& Harris Psychobiological category). Whereas seeing and hearing both achieved mean scores that placed them in the Body Independent category, feeling hungry obtained a mean discontinuity score in both samples, placing it in the Body Dependent category. Our results suggest that combining "seeing" and "hearing" with, for example, "knowing"' and "remembering'" may have generated more meaningful results.

\section{Conclusion}

The findings of this study contribute to debates within and between cognitive psychology and sociocultural anthropology on the cognitive foundations and patterns of cross-cultural 
recurrence and variation in mind-body thinking. The results suggest broad intersample agreement on which capacities, aptitudes, feelings, etc. participants judged to be dependent/independent on bodily function. The apparent facility to reason in terms of the autonomy of mental and biological function is not peculiar to Western populations, and there are significant cross-cultural parallels across the two samples with regard to where the line between "you" and "body" lies. There appears to be some divergence, however, concerning the roles of biological and physical properties in these assessments. Specifically, the Brazilian Amazonian population accorded considerable influence to physicality, but not biology, for items judged to be Body Dependent. UK participants judged both physicality and biology to importantly influence Body Dependent items and Body Independent items.

While we do not argue that our data support the position that person-body dualism is an intuitive default stance on the social world, we have presented findings that suggest that intuitions underpinning psycho-physical-biological reasoning for everyday traits, capacities, and feelings assume a broadly similar form cross-culturally. The cross-cultural similarities identified provide support for the view that the dualistic categories we term "person" and "body" may not be wholly culturally transmitted and are certainly not confined to the West. Although it is possible that the convergence in reasoning patterns is due to cultural factors largely independent of the conceptual constraints that characterize basic causal cognition, a more likely explanation, in the absence of any obvious reflective cultural beliefs and traditions that could inform responses to the full range of specific questions pertaining to this novel scenario, is that reasoning is informed and constrained by cognitive mechanisms that develop in broadly similar ways cross-culturally to deal with broadly similar social, biological, and physical worlds.

A rich empirical literature on the origins of intuitive causal reasoning about psychological, physical, and biological phenomena has demonstrated the early emergence of foundational causal cognition in these core areas. In the first year of life, for example, infants have a basic grasp of the principles of physical causation relevant to bounded physical objects, correctly expecting that objects move as cohesive wholes with spatiotemporal continuity, that they are subject to the laws of gravity, that they must be contacted to be launched, and that they cannot pass through other solid objects (Baillargeon, 1995; Spelke, 1994; Spelke, Breinlinger, Macomber, \& Jacobson, 1992; Spelke, Philips, \& Woodward, 1995). A wealth of evidence suggests that infants distinguish intentional agents from other kinds of physical entities on the basis of several cues, such as motion patterns (e.g., self-propelled vs. contactcaused motion), morphological features (e.g., presence vs. absence of eyes), patterns of interaction (e.g., contingent vs. noncontingent responsiveness), and purposeful action (e.g., presence vs. absence of goal-directedness) (e.g., Bloom, 2004; Murray \& Trevarthen, 1985; Rakison \& Poulin-Dubois, 2001; Wynn, 2008) applying different and appropriate expectations to these entities (Rakison \& Poulin-Dubois, 2001). Such early-emerging causal cognition may provide the foundation and scaffolding for further development of intuitive expectations and acquisition of cultural ideas about finer-grained within-domain and crossdomain interactions between psychological, psycho-physical, and psychobiological traits and bodily function. The causal principles that guide inferences, predictions, and expectations in one domain may be largely irrelevant to, and incommensurate with, the causal 
principles that guide reasoning in another domain. If so, many questions arise concerning how or whether reasoning becomes intuitively integrated over development or whether divergent principles are merely loosely juxtaposed under certain contexts, such as when reflectively assessing cross-domain interations within multi-domain entities (such as humans), experiences (such as bodily pain), and events (such as brain injury).

The significant differences between the two populations represented in this study further suggest that some aspects of mind-body reasoning are less constrained by the common workings of basic cognition and are therefore more susceptible to cultural tuning, for example, via exposure to divergent learning environments. The key differences concern (a) the relative importance of biology and physicality for body-dependent capacities, and (b) the conditions under which the body is perceived to be causally relevant to mental function. Specifically, we found that Brazilian participants appeared to be more resistant to the possibility of mind-body interactions than the UK participants. Prior and subsequent ethnographic research has not revealed why this may be the case. Further ethnographic research, data on children's developing reasoning about person-body phenomena using an adapted methodology, and the use of an ethnographically informed range of framing narratives, incorporating different priming conditions, may help to reveal the causal origins of these differences. The differences revealed by this preliminary investigation tentatively suggest that anthropologists may have both overestimated the influence of Descartes in sustaining mind-body dualism in the West and underestimated the influence of more recent scientific discoveries and theories in the fields of neurobiology and neuroscience more generally on thinking about mind-body interdependencies. Where this latter influence is particularly prevalent (e.g., via science education), conceptual resistance to more integrated, holistic mind-body reasoning might, under certain conditions, be weakened or overridden.

Further research is required to investigate developmental trajectories of person-body reasoning cross-culturally, the sources of cross-cultural recurrence and variation, the relationship between reflective and intuitive causal reasoning about person-body interactions and interdependencies, and the influence of cognitive and cultural factors in the spread of relevant concepts and practices. Regarding this final question, for example, we might expect to observe more frequently in the cross-cultural record concepts of ghosts who can see or feel happy than ghosts who can smell things, feel itchy, and so on. Future research might also investigate how cross-domain causal relations are conceptualized, looking specifically at the relationship between person-body reasoning and attributions of intentionality and responsibility. What assumptions guide reasoning about intentional control over different capacities thought to be more and less person-related or body-related? Perhaps the degree to which a capacity is thought to be Body Dependent correlates with assumptions about the degree to which it is impervious to mental influence. Based on the results of the present study, we suggest, for example, that people are more likely to attribute feeling happy to conscious control than feeling queasy. The current design did not allow for the inclusion of items that might be considered strictly bodily (e.g., whether one's hair still grows, or one's legs still work, etc.) as this may have engendered some confusion regarding the "you" being referred to in the question (i.e., the you that has left the body, or the body). Nevertheless, we would predict that conscious control is, in turn, more likely to be attributed some 
potential role in whether one feels queasy than in whether one's hair grows quickly or slowly. Relatedly, it would be of interest to ascertain whether degree of perceived conscious control correlates with degree of perceived responsibility. Systematic investigation in this area would potentially yield findings of importance for mental health education (e.g., regarding the biological underpinnings of depression) and professional training (see Miresco \& Kirmayer, 2006).

Finally, we sought to assess the validity of mental state categories established in current relevant literature on afterlife beliefs, and to develop novel categories for use in future research on mind-body dualism. The framing narrative of the Disembodied Condition closely resembles the basic structure of widespread concepts of disembodied agents, characteristic of religious concepts but also common in popular fiction and film, and children's imaginary worlds. For example, concepts of afterlife and ancestor spirits, reincarnation, spirit possession, soul flight, mind swaps, imaginary companions, and so on, share a common conceptual commitment to the same radical disconnect between person and body that we invited participants to imagine. The design of our study therefore allowed us to assess the validity of mental state categories used in the existing cognitive psychological literature that are purported to carve up the domain according to the degree to which they are perceived to be tied to bodily function.

In the UK sample, all categories previously used in the literature failed to cohere, with one or more items obtaining mean continuity rankings significantly different to one or more items in the group. In the Brazil sample, only the Bering and Bjorklund Epistemic category achieved a nonsignificant result (suggesting that items in this category were ranked similarly by participants in terms of their perceived anchoring to bodily function). We have presented suggestions for new, validated Body Dependent and Body Independent categories for future research on person-body reasoning.

In conclusion, the cross-cultural study reported offers the first systematic, cross-cultural analysis of person-body reasoning across a broad range of capacities, provides firm evidential grounds for the refinement of theory and method in future cognitive psychological and anthropological research, and suggests numerous lines of potential further inquiry on the emergence and spread of patterns of recurrence and variation in mind-body dualism in particular, and person-body reasoning generally.

\section{Notes}

1. BBC News. "Donor organ 'personality' worry." Available at: http://news.bbc.co. uk/1/hi/8084936.stm. Accessed June 5, 2009.

2. Rural, non-indigenous Brazilian Amazon communities have received relatively little attention in the ethnographic literature. Notable contributions to the scarce ethnography of these "people without an official ethno" (Nugent, 1993, p. xviii) are Harris, 1998, 2000; Nugent, 1993, 1997; Pace, 1998; Wagley, 1976 [1953].

3. Four further items were included in the questionnaire but subsequently dropped from analysis due to evidence of comprehension problems with the Portuguese translation 
at the time of administering the questionnaire to the Brazilian sample. The four items were feel bored, forget things, feel tingly, feel jealous.

4. UK/Brazil mean scores were 5.14/5.32 (Disembodied), 4.79/4.92 (Rock), and 3.71/4.29 (Plant)

\section{Acknowledgements}

This research was funded by the John Templeton Foundation. We are grateful to Andrezza Barbosa, Matt Barnard, Renato Cohen, Lúcia Coutinho, Ann Cowie, Jean Gê, and Tenelle Porter for assistance with data collection, to Matt Grove, Claire White, and Harvey Whitehouse for advice and feedback on data analysis, and to Nancy Nersessian and four anonymous reviewers for their very helpful suggestions and comments. Brazilian Portuguese translations of interview materials are available on request.

\section{References}

Ahn, W., Proctor, C. C., \& Flanagan, E. H. (2009). Mental health clinicians' beliefs about the biological, psychological, and environmental bases of mental disorders. Cognitive Science, 33(2), 147-182.

Astuti, R. (2001). Are we all natural dualists? A cognitive developmental approach. Journal of the Royal Anthropological Institute, 7, 429-447.

Astuti, R., \& Harris, P. L. (2008). Understanding mortality and the life of the ancestors in rural Madagascar. Cognitive Science, 32, 713-740.

Baillargeon, R. (1995). A model of physical reasoning in infancy. In C. Rovee-Collier \& L. P. Lipsitt (Eds.), Advances in infancy research, Vol. 9, (pp. 305-371). Norwood, NJ: Ablex.

Bering, J. M., \& Bjorklund, D. F. (2004). The natural emergence of reasoning about the afterlife as a developmental regularity. Developmental Psychology, 40, 217-233.

Bering, J. M., Hernández Blasi, C., \& Bjorklund, D. F. (2005). The development of 'afterlife' beliefs in religiously and secularly schooled children. British Journal of Developmental Psychology, 23, 587-607.

Bloom, P. (2004). Descartes' baby: How child development explains what makes us human. New York: Basic Books.

Cohen, E. (2007). The mind possessed: The cognition of spirit possession in an afro-brazilian religious tradition. New York: Oxford University Press.

Cohen, E. (2008). What is spirit possession? Defining, comparing, and explaining two possession forms. Ethnos, 73(1), 101-126.

Cohen, E., \& Barrett, J. (2008a). When minds migrate: Conceptualizing spirit possession. Journal of Cognition and Culture, 8, 23-48.

Cohen, E., \& Barrett, J. (2008b). Conceptualizing spirit possession: Ethnographic and experimental evidence. Ethos, 36, 245-266.

Frankel, S. (1986). The huli response to illness. Cambridge, England: Cambridge University Press.

Harris, M. (1998). "What is means to be Caboclo"': Some critical notes on the construction of Amazonian caboclo society as an anthropological object. Critique of Anthropology, 18, 83-95.

Harris, M. (2000). Life on the Amazon: The anthropology of a Brazilian peasant village. New York: Oxford University Press.

Harris, P. L., \& Giménez, M. (2005). Children's acceptance of conflicting testimony: The case of death. Journal of Cognition and Culture, 5, 143-164. 
Hatano, G., \& Inagaki, K. (1994). Young children's naïve theory of biology. Cognition, 50, 171-188.

Hodge, M. (2008). Descartes' mistake: How afterlife beliefs challenge the assumption that humans are intuitive Cartesian substance dualists. Journal of Cognition and Culture, 8, 387-415.

Inagaki, K., \& Hatano, G. (1993). Young children's understanding of the mind-body distinction. Child Development, 64, 1534-1549.

Ingold, T. (1991). Becoming persons. Consciousness and sociality in human evolution. Cultural Dynamics, 4, $355-378$.

Kuhlmeier, V. A., Bloom, P., \& Wynn, K. (2004a). Do 5-month-old infants see humans as material objects? Cognition, 94, 95-103.

Kuhlmeier, V. A., Bloom, P., \& Wynn, K. (2004b). People v. objects: A reply to Rakison and Cicchino. Cognition, 94, 109-112.

Lambek, M. (1998). Body and mind in mind, body and mind in body: Some anthropological interventions in a long conversation. In M. Lambek \& M. Strathern (Eds.), Bodies and persons: Comparative perspectives from Africa and Melanesia (pp. 103-123). Cambridge, England: Cambridge University Press.

Lewis, G. (2004). Knowledge of illness in Sepik society: A study of the Gnau, New Guinea (LSE Monographs on Social Anthropology). Oxford, England: Berg Publishers.

McCallum, C. (1996). The body that knows: From Cashinahua epistemology to a medical anthropology of Lowland South America. Medical Anthropology Quarterly, 10, 347-372.

Miresco, M. J., \& Kirmayer, L. J. (2006). The persistence of mind-brain dualism in psychiatric reasoning about clinical scenarios. American Journal of Psychiatry, 163(5), 913-918.

Murray, L., \& Trevarthen, C. (1985). Emotional regulation of interactions between two-month-olds and their mothers. In T. M. Field \& N. A. Fox (Eds.), Social perception in infants (pp. 177-197). Norwood, NJ: Ablex.

Notaro, P., Gelman, S., \& Zimmerman, M. A. (2001). Children's understanding of psychogenic bodily reactions. Child Development, 72(2), 444-459.

Notaro, P., Gelman, S., \& Zimmerman, M. A. (2002). Biases in reasoning about the consequences of psychogenic bodily reactions: Domain boundaries in cognitive development. Merrill-Palmer Quarterly, 48, 427449.

Nugent, S. (1993). Amazonian Caboclo society: An essay on invisibility and peasant economy. Oxford, England: Berg.

Nugent, S. (1997). The coordinates of identity: At play in the fields of culture. Critique of Anthropology, 17, $33-51$.

Pace, R. (1998). The struggle for Amazon Town: Gurupá revisited. Boulder, CO: Lynne Rienner Publishers, Inc.

Rakison, D. H., \& Cicchino, J. (2004). Is an infant a people person? Cognition, 94, 105-107.

Rakison, D. H., \& Poulin-Dubois, D. (2001). Developmental origin of the animate-inanimate distinction. Psychological Bulletin, 127(2), 209-228.

Rival, L. (1998). Androgynous parents and guest children: The Huaorani couvade. Journal of the Royal Anthropological Institute, 4, 619-642.

Saxe, R., Tzelnic, T., \& Carey, S. (2006). Five-month-old infants know humans are solid, like inanimate objects. Cognition, 101, B1-B8.

Scheper-Hughes, N., \& Lock, M. (1987). The mindful body: A prolegomenon to future work in medical anthropology. Medical Anthropology Quarterly (NS), 1, 6-41.

Schultz, L., Bonawitz, E. B., \& Griffiths, T. L. (2007). Can being scared cause tummy aches? Naïve theories, ambiguous evidence, and preschoolers' causal inferences. Developmental Psychology, 43(5), 1124-1139.

Spelke, E. (1994). Initial knowledge: Six suggestions. Cognition, 50, 443-447.

Spelke, E. S., Breinlinger, K., Macomber, J., \& Jacobson, K. (1992). Origins of knowledge. Psychological Review, 99, 605-632.

Spelke, E. S., Philips, A., \& Woodward, A. L. (1995). Infants' knowledge of object motion and human action. In D. Sperber, D. Premack \& A. J. Premack (Eds.), Causal cognition (pp. 150-184). Oxford, England: Clarendon.

Strathern, A. (1994). Keeping the body in mind. Social Anthropology, 2, 43-53. 
Strathern, A. (1996). Body thoughts. Ann Arbor, MI: University of Michigan Press.

Vilaça, A. (2002). Making kin out of others in Amazonia. Journal of the Royal Anthropological Institute, $8,347-365$.

Wagley, C. 1976 [1953]. Amazon Town: A study of man in the tropics. New York: Oxford University Press.

Wellman, H., \& Johnson, C. (2008). Developing dualism: From intuitive understanding to transcendental ideas. In A. Antonietti, A. Corradini \& E. Jonathan Lowe (Eds.), Psycho-physical dualism today: An interdisciplinary approach (pp. 3-36). Lanham, MD: Lexington Books.

Wynn, K. (2008). Some innate foundations of social and moral cognition. In P. Carruthers, S. Laurence \& S. Stich (Eds.), The innate mind: Foundations and the future (pp. 330-347). New York: Oxford University Press. 